\title{
Syndromic Surveillance Based on Emergency Visits: A Reactive Tool for Unusual Events Detection
}

\author{
Pascal Vilain*1, Arnaud Bourdé2, Pierre-Jean Marianne dit Cassou ${ }^{3}$, Yves Jacques- \\ Antoine $^{4}$, Philippe Morbidelli ${ }^{5}$ and Laurent Filleul ${ }^{1}$
}

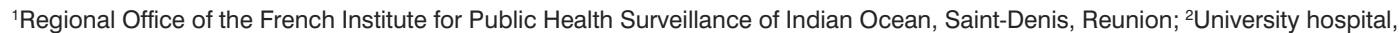
Saint-Denis, Reunion; ${ }^{3}$ University hospital, Saint-Pierre, Reunion; ${ }^{4}$ Hopital center, Saint-Benoît, Reunion; ${ }^{5}$ Hopital center, Saint-Paul, Reunion

\section{Objective}

To show with examples that syndromic surveillance system can be a reactive tool for public health surveillance.

\section{Introduction}

The late health events such as the heat wave of 2003 showed the need to make public health surveillance evolve in France. Thus, the French Institute for Public Health Surveillance has developed syndromic surveillance systems based on several information sources such as emergency departments (1). In Reunion Island, the chikungunya outbreak of 2005-2006, then the influenza pandemic of 2009 contributed to the implementation and the development of this surveillance system (2-3). In the past years, this tool allowed to follow and measure the impact of seasonal epidemics. Nevertheless, its usefulness for the detection of minor unusual events had yet to be demonstrated.

\section{Methods}

In Reunion Island, the syndromic surveillance system is based on the activity of six emergency departments. Two types of indicators are constructed from collected data:

- Qualitative indicators for the alert (every visit whose diagnostic relates to a notifiable disease or potential epidemic disease);

- Quantitative indicators for the epidemic/cluster detection (number of visits based on syndromic grouping).

Daily and weekly analyses are carried out. A decision algorithm allows to validate the signal and to organize an epidemiological investigation if necessary.

\section{Results}

Each year, about 150000 visits are registered in the six emergency departments that is 415 consultations per day on average. Several unusual health events on small-scale were detected early.

In August 2011, the surveillance system allowed to detect the first autochthonous cases of measles, a few days before this notifiable disease was reported to health authorities (Figure 1). In January 2012, the data of emergency departments allowed to validate the signal of viral meningitis as well as to detect a cluster in the West of the island and to follow its trend. In June 2012, a family foodborne illness was detected from a spatio-temporal cluster for abdominal pain by the surveillance system and was confirmed by epidemiological investigation (Figure 2).

\section{Conclusions}

Despite the improvement of exchanges with health practitioners and the development of specific surveillance systems, health surveillance remains fragile for the detection of clusters or unusual health events on small scale. The syndromic surveillance system based on emergency visits has proved to be relevant for the identification of signals leading to health alerts and requiring immediate control measures. In the future, it will be necessary to develop these systems (private practitioners, sentinel schools) in order to have several indicators depending on the degree of severity.

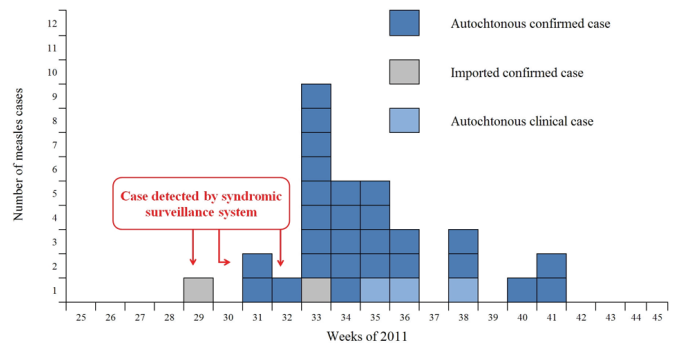

Figure 1. Epidemic curve of measles cases

\begin{tabular}{|c|l|l|l|l|l|l|}
\hline Date & ICD-10 & \multicolumn{1}{c|}{ Label } & \multicolumn{3}{c|}{ Time Gender Zip code } \\
\hline 17/06/2012 R10 & Abdominal and pelvic pain & $2: 15$ & 39 & F & 97431 \\
\hline 17/06/2012 R10 & Abdominal and pelvic pain & $2: 17$ & 1 & F & 97431 \\
\hline 17/06/2012 R10 & Abdominal and pelvic pain & $2: 18$ & 12 & F & 97431 \\
\hline 17/06/2012 R10 & Abdominal and pelvic pain & $2: 20$ & 17 & M & 97431 \\
\hline 17/06/2012 R10.2 & Pelvic and perineal pain & $0: 15$ & 27 & F & 97470 \\
\hline 17/06/2012 R10.2 & Pelvic and perineal pain & $3: 33$ & 17 & F & 97412 \\
\hline
\end{tabular}

Figure 2. Line-list of patient characteristics in an abdominal pain cluster.

\section{Keywords}

Syndromic surveillance; Unusual event detection; Reunion Island

\section{Acknowledgments}

We are thankful to all the practitioners of emergency departments.

\section{References}

1. Josseran L, Nicolau J, Caillère N, Astagneau P, Brücker G. Syndromic surveillance based on emergency department activity and crude mortality: two examples. Euro Surveill. 2006;11(12):225-9.

2. D’Ortenzio E, Do C, Renault P, Weber F, Filleul L. Enhanced influenza surveillance on Réunion Island (southern hemisphere) in the context of the emergence of influenza A(H1N1)v. Euro Surveill. 2009;14(26). pii: 19257.

3. Filleul L, Durquety E, Baroux N, Chollet P, Cadivel A, Lernout T. The development of non-specific surveillance in Mayotte and Reunion Island in the contexte of the epidemic influenza A(H1N1) 2009 [Article in French]. Bull EpidemiolHebd. 2010:283.

*Pascal Vilain

E-mail: pascal.vilain@ars.sante.fr 